\title{
Intense High-Contrast Femtosecond $K$-Shell X-Ray Source from Laser-Driven Ar Clusters
}

\author{
L. M. Chen, ${ }^{1, *}$ F. Liu, ${ }^{1}$ W. M. Wang, ${ }^{1}$ M. Kando, ${ }^{2}$ J. Y. Mao, ${ }^{1}$ L. Zhang, ${ }^{1}$ J. L. Ma, ${ }^{1}$ Y. T. Li, ${ }^{1}$ S. V. Bulanov, ${ }^{2}$ T. Tajima, ${ }^{2}$ \\ Y. Kato, ${ }^{2}$ Z. M. Sheng, ${ }^{1,3}$ Z. Y. Wei, ${ }^{1}$ and J. Zhang ${ }^{1,3, \dagger}$ \\ ${ }^{1}$ Beijing National Laboratory of Condensed Matter Physics, Institute of Physics, CAS, Beijing 100080, China \\ ${ }^{2}$ Advanced Photon Research Center, Japan Atomic Energy Agency, 8-1 Umemidai Kizugawa, Kyoto 619-0215, Japan \\ ${ }^{3}$ Department of Physics, Shanghai Jiao Tong University, Shanghai 200240, China
}

(Received 23 January 2010; published 27 May 2010)

\begin{abstract}
Bright Ar quasimonochromatic $K$-shell x ray with very little background has been generated using an Ar clustering gas jet target irradiated with a 30 fs ultrahigh-contrast laser, with a measured flux of $2.2 \times$ $10^{11}$ photons/J into $4 \pi$. This intense $\mathrm{X}$-ray source critically depends on the laser contrast and intensity. The optimization of source output with interaction length is addressed. Simulations point to a nonlinear resonant mechanism of electron heating during the early stage of laser interaction, resulting in enhanced $\mathrm{x}$-ray emission. The $\mathrm{x}$-ray pulse duration is expected to be only $10 \mathrm{fs}$, opening the possibility for singleshot ultrafast $\mathrm{keV} \mathrm{x}$-ray imaging applications.
\end{abstract}

Synchrotron light sources have proven very useful for a wide range of investigations. However, it is inappropriate for some applications because of its large cost and experimental footprint, relatively long pulse structure, and polychromaticity. Hard-x-ray emission from femtosecond (fs) laser-produced plasmas has been extensively studied in past years, including, for example, Refs. [1-5]. Such hard-x-ray sources are of interest for a number of imaging applications [6-9]. This kind of intense and ultrafast hard$\mathrm{x}$-ray source can provide an alternative to synchrotron radiation sources due to its compactness, subpicosecond pulse duration, and monochromaticity, making it practical for widespread use in labs and hospitals.

Presently, there remain serious obstacles limiting laserdriven hard-x-ray sources in imaging applications, e.g., the limited $K \alpha$ x-ray conversion efficiency $\left(\sim 10^{-5}\right)$ and the spectrum "contrast ratio" of $K \alpha$ to the continuum background [10]. The hard-x-ray emission produced by high intensity laser-solid interactions relies on hot electrons [11], which penetrate into the solid target and produce hard-x-ray emission via $K$-shell ionization and bremsstrahlung. Typically, the x-ray continuum in such a spectrum is strong and usually contains $90 \%$ of total x-ray energy with photon energies $>1 \mathrm{keV}$ [4]. In the worst case, the highenergy $\mathrm{x}$-ray tail will greatly reduce the subject contrast of in-line radiography [8]. The microdroplet is another candidate $\mathrm{x}$-ray source material, with similar conversion efficiency as a solid target, but the strong x-ray energetic tail limits its application for imaging for the same reason. $\mathrm{X}$-ray sources from laser-cluster interaction, usually using the normal contrast laser pulse (with main pulse to prepulse ratio $<10^{6}$ ) and large size clusters, suffer from low conversion efficiency, which has been found to be $\sim 10^{-7}$ for $\mathrm{Ar}$ and $\mathrm{Kr}$ clusters [12,13]. Again, its spectrum also has a strong bremsstrahlung continuum, which reduces the $K$-shell line emission contrast. Therefore, the laser-driven hard-x-ray source with more intense flux and higher spec- trum contrast ratio needed for imaging application is the subject of many current investigations.

In this Letter, we present generation of intense $\mathrm{Ar}$ $K$-shell $\mathrm{x}$ rays with very weak continuum background using a small cluster target irradiated with a high-contrast fs laser pulse. The intensity of the $\operatorname{Ar} K$-shell emission in the forward direction has been measured to be $1.1 \times$ $10^{4}$ (photons $/ \mathrm{mrad}^{2}$ )/pulse, enough for single-shot $\mathrm{x}$-ray imaging. A nonlinear resonant mechanism is found to predominate in the case of high-contrast fs laser interaction with nanometer-size clusters which drive electrons very efficiently in the 10 fs time scale, enhancing $x$-ray emission.

The experiments are carried out by using a facility, which includes a $10 \mathrm{~Hz}, 800 \mathrm{~mJ}$ Ti:sapphire laser working at the center wavelength $\lambda=800 \mathrm{~nm}$. The pulse with duration $\tau_{0}=28 \mathrm{fs}$ is focused with an $f / 13$ off-axis parabola (OAP) onto a focal spot of size $w_{0}=16 \mu \mathrm{m}$. In the focal region the laser average intensity is $I=1.6 \times$ $10^{18} \mathrm{~W} / \mathrm{cm}^{2}$. With the help of optical parametric chirped pulse amplification, the laser pulse contrast, compared to its nanosecond prepulse, has been improved to $10^{9}$. A supersonic pulsed gas (Ar) jet is used, which is $3 \mathrm{~mm}$ in diameter at the exit. A strong magnet is placed after the nozzle to expel electrons. A filtered 16-bit single-photoncounting CCD (Roper Scientific) is located in the laser propagation direction to detect the $\mathrm{x}$-ray flux with a photon energy $>0.8 \mathrm{keV}$. The calibration shows that the resolving power of the CCD as a dispersionless spectrometer at $\sim 3 \mathrm{keV}$ is about 20. A knife edge is introduced to measure the $\mathrm{x}$-ray source size. A probe beam is used for detecting the shadowgraph. The cluster size is estimated by employing the Hagena scaling law [14]. An average size of $\sim 8 \mathrm{~nm}$ in diameter is assumed at a stagnation pressure of $4 \mathrm{MPa}$.

Figure 1 shows the Ar x-ray spectrum obtained in a single shot. Compared to the $\mathrm{x}$-ray spectrum from a solid $\mathrm{Cu}$ target with similar laser parameters, the $\operatorname{Ar} K$-shell 
emission shows much higher contrast (lines to continuous background ratio). The amplitude of the continuum part of the spectrum is about $1 \%$ compared to the peak of the $K$ emission line. The integrated $K$-shell photon number is more than $95 \%$ of all the photons in the spectrum, whereas in previous reports it is only $\sim 10 \%$ in the case of a solid target [4] and larger-size cluster target [13] irradiated by normal contrast laser pulses. The $K$-shell x-ray conversion efficiency from laser energy is about $10^{-4}$, which is over 100 times higher than previous observations $[12,13]$. It should be emphasized that the x-ray flux (and spectral distribution) depends critically on the laser contrast. The flux is reduced by 2 orders of magnitude if the laser pulse contrast decreases from $10^{9}$ to $10^{7}$ with constant laser pulse energy. Preexpansion of a solid-density cluster by the laser prepulse leads to ineffective heating of electrons, as described below, and results in the decrease of x-ray flux in this case. We also observe that this high-contrast, quasimonochromatic structure for the $K$-shell emission disappears if energetic electrons are generated, unlike the case in Ref. [15]. The x-ray continuous background becomes a biMaxwellian distribution [16], dramatically reducing the $\mathrm{x}$-ray line contrast.

By changing laser and interaction conditions, the Ar $\mathrm{x}$-ray emission can be optimized. The $\mathrm{x}$-ray emission increases linearly by increasing the gas backing pressure $(P)$ and then saturates when $P>4 \mathrm{MPa}$, whereas the cluster size continues to increase beyond $4 \mathrm{MPa}$. A threshold is observed for x-ray emission as a function of laser pulse intensity, as shown in the inset of Fig. 1. When the laser pulse average intensity is higher than $10^{16} \mathrm{~W} / \mathrm{cm}^{2}$, the $\mathrm{x}$-ray flux increases steeply and reaches a maximum at $I \geq$ $10^{17} \mathrm{~W} / \mathrm{cm}^{2}$. A more interesting phenomenon is that the detected photon intensity is a function of the nozzle displacement away from laser focal spot, as demonstrated in

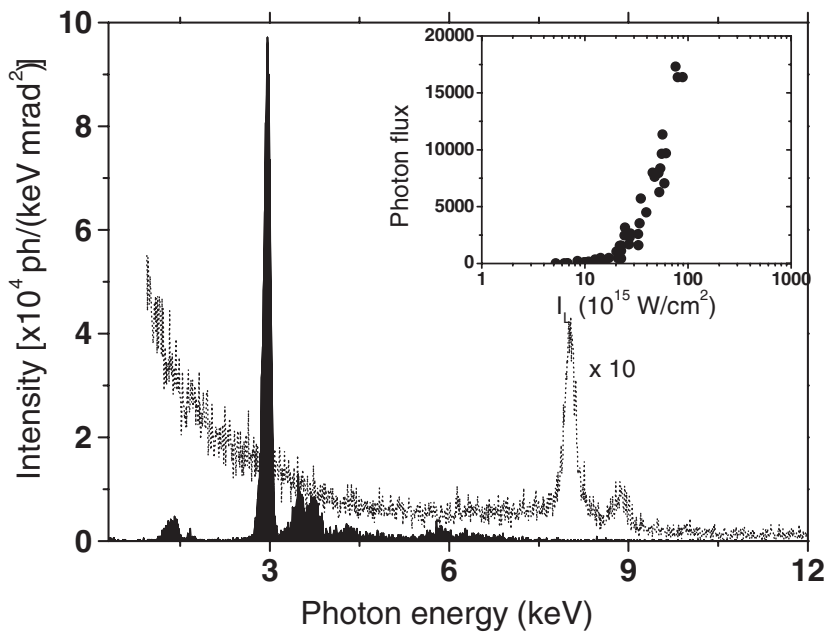

FIG. 1. X-ray spectrum of Ar when the laser beam was focused on the cluster target. The $\mathrm{x}$-ray spectrum of a solid $\mathrm{Cu}$ target is also shown (dotted line) with $\times 10$ times in amplitude. The inset shows the photon flux dependence upon laser intensity.
Fig. 2. If the laser focuses on the edge of the plasma, energetic electrons are generated and the $K$-shell x-ray flux is very unstable shot to shot. As the nozzle moves away from the laser focal spot, the photon flux decreases at first, then increases, reaching a maximum of $1.1 \times$ $10^{4}$ photons $/ \mathrm{mrad}^{2}$ when the nozzle moves close to the OAP $\sim 6 \mathrm{~mm}$. In our experiment, the total flux reached $2.2 \times 10^{11}$ photons/J into $4 \pi$, and the $K$-shell x-ray generation was very stable. The laser intensity on the edge of plasma is reduced to $5 \times 10^{16} \mathrm{~W} / \mathrm{cm}^{2}$, which is similar to our previous observation using a lower powered laser [16]. The plasma volume obtained in the shadowgraph (inset of Fig. 2) for the case of best focus and defocus demonstrates that a long and straight laser channel leads to the enhancement of the $K$-shell emission. As seen in the shadowgraph, the plasma channel with diameter $60 \mu \mathrm{m}$ in the defocus case is much longer than the case of best focus, providing a much larger interaction volume. The $\mathrm{x}$-ray source sizes detected via the knife edge technique in the case of $6 \mathrm{~mm}$ defocus are $\sim 20 \mu \mathrm{m}$ in FWHM, smaller than the laser focal spot size. If the nozzle displacement is less than $5 \mathrm{~mm}$, the electrons will be accelerated and produce strong $\gamma$-ray background.

Simulations using a 2D fully electromagnetic particlein-cell code have been performed, where a linearly polarized laser pulse with $\sin ^{2}$ pulse envelope is launched along the $+x$ direction onto a cluster with $10 \mathrm{~nm}$ diameter and $10 n_{\text {cr }}$ (critical density). Figure 3(a) shows snapshots of the electron distribution profile at various times with $I=1 \times$ $10^{17} \mathrm{~W} / \mathrm{cm}^{2}$. At the early stage of the laser pulse, electrons are driven by the laser electric field and quiver along the laser polarization direction ( $y$ direction) in each laser period; the early stage energy and phase spectra $\left(p_{x}, p_{y}\right)$ are

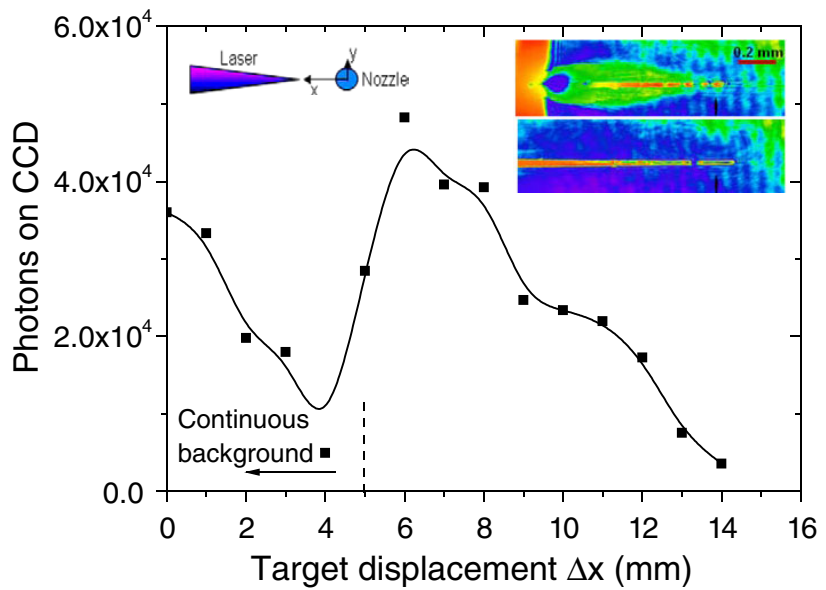

FIG. 2 (color online). Channeled emission characteristics. The $K \alpha$ photon emission dependence as a function of the target displacement $\Delta x$ from the best laser focus. The upper right inset shows the shadowgraph obtained in the case of the nozzle located on the focal spot position (short channel) and a position $\Delta x=+6 \mathrm{~mm}$ (long channel). The positive displacement means the nozzle moves closer to the OAP. 

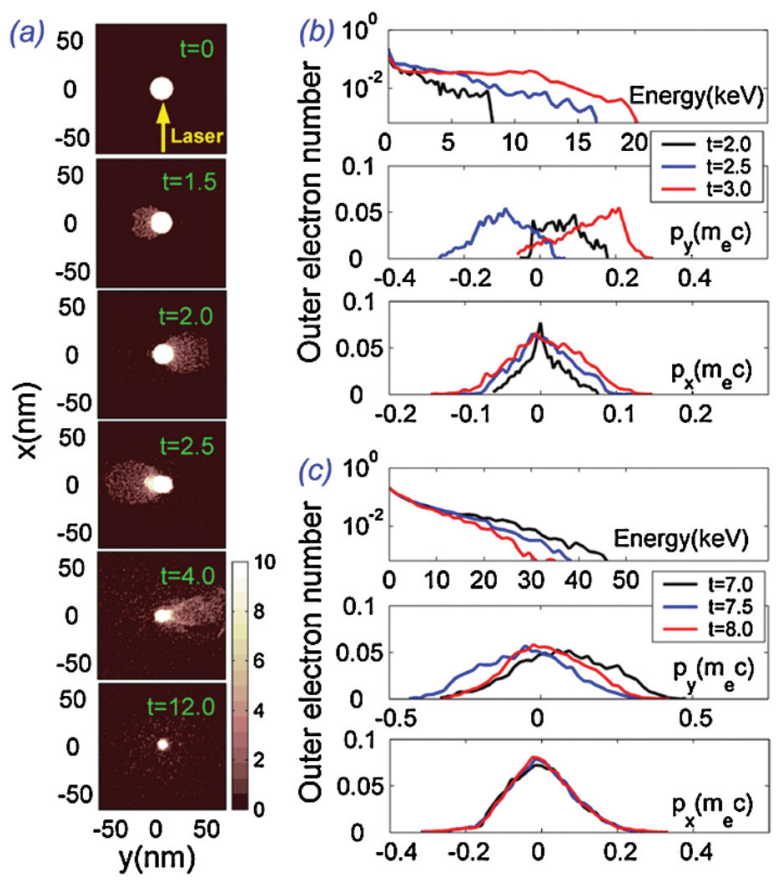

FIG. 3 (color). Simulation snapshots of the electron density distribution (a) of cluster at various times (with unit of laser optical cycle) in laser intensity $1 \times 10^{17} \mathrm{~W} / \mathrm{cm}^{2}$. The energy spectra and phase spectra $\left(P_{x}, P_{y}\right)$ of outer electrons at various time are shown on the early stage of laser pulse (b) and on the peak of pulse (c).

shown in Fig. 3(b). Figure 4(a) shows the electron number of inner electrons generally decreasing with time, but for each half optical cycle, this is modulated by part of the electron density returning to the cluster boundary, i.e., electron quiver. This phenomenon is defined as the nonlinear resonant (NLR) electron heating mechanism [17]. These electrons, with quiver energy shown as these small spikes on the curve in Fig. 4(d), will pass through the cluster and stimulate $K$-shell ionization to produce $\mathrm{x}$ rays when the electron energy is larger than the Ar ionization threshold. It is important to point out that the quiver electrons possess non-Maxwellian electron energy distribution in the early stage [Fig. 3(b)], which is optimal to stimulate $\operatorname{Ar} K$-shell x ray with much less continuous background. However, this quiver phenomenon dampens at the pulse latter stage because an electron cloud forms from escaping electrons around the cluster and this compensates the effect of the laser electric field, as seen in Fig. 3(a) and 3(c). Therefore, we conclude that the $K$-shell $\mathrm{x}$-ray photon generation arises from electrons quivering in the early stage of intense laser fields. Electrons possess a quiver energy $E_{q}[\mathrm{eV}]=e^{2} E^{2} /\left(2 m_{e} \omega_{0}^{2}\right)=9.3 \times$ $10^{-14} I\left[\mathrm{~W} / \mathrm{cm}^{2}\right] \lambda^{2}[\mu \mathrm{m}]$. For $I=10^{17} \mathrm{~W} / \mathrm{cm}^{2}, \quad E_{q} \sim$ $6 \mathrm{keV}$. This is high enough to stimulate $\operatorname{Ar} K$-shell photons at energies $E_{k} \sim 3 \mathrm{keV}$ and considering the cross section of $\mathrm{Ar}$ is near optimal [18]. It also explains that there is a laser threshold intensity to stimulate $K$-shell x-ray emis-
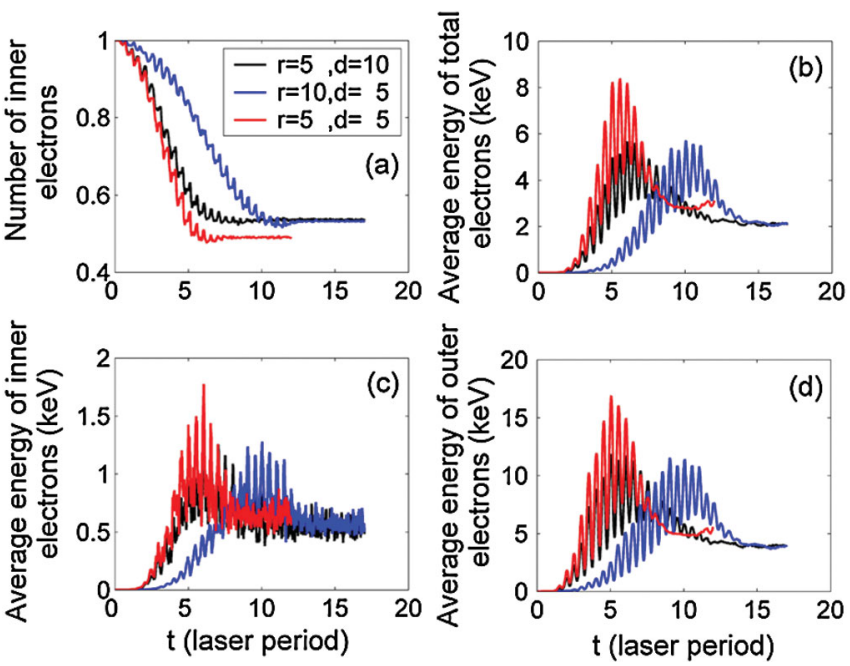

FIG. 4 (color). Laser pulse shape dependent. The simulation temporal distribution of number of inner electrons (a), energy of total electrons (b), energy of inner electrons (c), and energy of outer electrons (d). The laser pulse with rising (down) edge 5/5 (red line), 5 (10) (black line), 10 (5) (blue line) are used.

sion in Fig. 1. A more powerful facility is necessary, with higher laser intensity, to stimulate higher energy $K$-shell $\mathrm{x}$-ray emission such as $\mathrm{Kr}\left(E_{k} \sim 12.6 \mathrm{keV}\right)$. In Fig. 4(d), only 4-5 optical cycles, with spikes higher than $5 \mathrm{keV}$, can produce $K$-shell x ray which corresponds to the duration about 10 fs. Considering the $\operatorname{Ar} K$-shell vacancy lifetime $\sim 4.8$ fs [19], we conclude that the $K$-shell x-ray pulse duration is as short as $10 \mathrm{fs}$, and so this is a demonstration of an ultrashort hard-x-ray source. On the other hand, the cluster inner electrons obtain energy much lower than $E_{k}$, which does not then contribute to $K$-shell ionization in our case, as shown in Fig. 4(c). This rules out the linear resonant (LR) heating [20] and the long-term ionization mechanisms of laser-cluster interactions that dominate in the case of using a normal contrast laser.

The transfer from NLR to LR is shown experimentally by using the high-contrast laser. By changing the distance between the compressor gratings away from the "zero" position at constant laser energy, the incomplete compensation of the accumulated phase nonlinearities results in negatively (positively) skewed pulses having a gentle (steep) rise time [10]. In the case of laser irradiation with intensity $1 \times 10^{17} \mathrm{~W} / \mathrm{cm}^{2}$, dependence of the $\mathrm{x}$-ray flux on laser pulse duration in the negatively skewed and positively skewed cases shows different behavior. As shown in Fig. 5(a), the flux using positively skewed pulses drops less precipitously than the case of negatively skewed pulses. Simulations in Fig. 4(d) using different pulse shapes successfully reproduced this phenomenon. The laser intensity is still strong enough to drive electron heating efficiently when laser pulse duration slightly expands, stimulating the NLR. Positively skewed pulses drive higher spikes than the case of negatively pulsed irradiation; even 

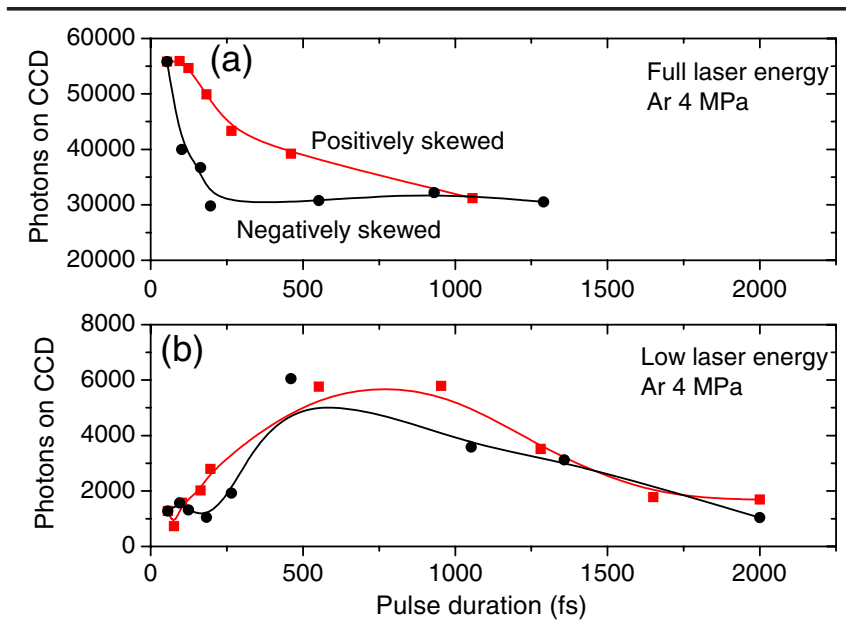

FIG. 5 (color online). Ar $K$-shell x-ray emission as a function of laser pulse width with negatively skewed pulse shape (red line) and positively skewed pulse shape (black line) for (a) a full energy pulse and (b) low-energy pulse irradiation.

the latter have weaker spikes that are not strong enough to efficiently stimulate $\operatorname{Ar} K$-shell ionization. However, when the laser intensity drops to $1 \times 10^{16} \mathrm{~W} / \mathrm{cm}^{2}$, the laser electric field cannot heat electrons effectively in the fs time scale. The x-ray flux is dramatically reduced, as shown in Fig. 5(b). In this case, the cluster needs some time, usually $500 \mathrm{fs}-1 \mathrm{ps}$ [20], to expand to the critical density for stimulating the LR mechanism. Just as expected, the x-ray flux in Fig. 5(b) reaches a peak at that laser pulse duration, which is indicative of actively controlling the evolution of the cluster heating mechanism from NLR to LR regimes using the high-contrast laser.

In summary, we have presented a method for generation of intense $\operatorname{Ar} K$-shell x rays with very weak background using a cluster target irradiated with an intense fs laser pulse. The intensity of the Ar x-ray emission has been measured to be $1.1 \times 10^{4}\left(\mathrm{photons} / \mathrm{mrad}^{2}\right) /$ pulse, which is sufficient to perform phase contrast $\mathrm{x}$-ray imaging with a single laser shot using an imaging plate $20 \mathrm{~cm}$ away from the source [16]. Nonlinear resonant heating is believed to be the dominant mechanism in the case of high-contrast fs laser interacting with nanometer-size clusters; here in this regime, electrons are efficiently driven in a $10 \mathrm{fs}$ time scale, producing enhanced $\mathrm{x}$-ray emission with duration about 3 orders shorter than that of typical pulses produced by synchrotron sources. Together with the measured source size of $20 \mu \mathrm{m}$, the peak brightness of the radiation is estimated to be $\sim 2 \times 10^{21}$ photons $/\left(\mathrm{s} \mathrm{mm}^{2} \mathrm{mrad}^{2}\right)$, which is comparable to the peak brightness of the third generation synchrotron radiation sources. This ultraintense, monochromatic and fs duration hard-x-ray source may make possible "single-shot" laser-driven $\mathrm{x}$-ray ultrafast applications.

We thank L. T. Hudson and J. F. Seely for fruitful discussions. This work was supported by the NSFC (Grants No. 60878014, No. 10974249, No. 10735050, No. 10925421, and No. 10734130), National Basic Research Program of China (973 Program) (Grant No. 2007CB815102). and the National High-Tech 863 program.

*Imchen@aphy.iphy.ac.cn †jzhang1@sjtu.edu.cn

[1] M. Yoshida, Y. Fujimoto, Y. Hironaka, K. G. Nakamura, K. Kondo, M. Ohtani, and H. Tsunemi, Appl. Phys. Lett. 73, 2393 (1998).

[2] Th. Schlegel, S. Bastiani, L. Grémillet, J.-P. Geindre, P. Audebert, J.-C. Gauthier, E. Lefebvre, G. Bonnaud, and J. Delettrez, Phys. Rev. E 60, 2209 (1999).

[3] M. Schnurer, R. Nolte, A. Rousse, G. Grillon, G. Cheniaux, M.P. Kalachnikov, P. V. Nickles, and W. Sanders, Phys. Rev. E 61, 4394 (2000).

[4] J. Yu, Z. Jiang, J. C. Kieffer, and A. Krol, Phys. Plasmas 6, 1318 (1999).

[5] Ch. Reich, P. Gibbon, I. Uschmann, and E. Forster, Phys. Rev. Lett. 84, 4846 (2000).

[6] C. Tillman, A. Persson, C. G. Wahlstrom, S. Svanberg, and K. Herrlin, Appl. Phys. B 61, 333 (1995).

[7] C. L. Gordon III, G. Y. Yin, B. E. Lemoff, P. M. Bell, and C. P. J. Barty, Opt. Lett. 20, 1056 (1995).

[8] A. Krol et al., Med. Phys. 24, 725 (1997).

[9] J. C. Kieffer, A. Krol, Z. Jiang, C. C. Chamberlain, E. Scalzetti, and Z. Ichalalene, Appl. Phys. B 74, S75 (2002), and references therein.

[10] L. M. Chen et al., Phys. Rev. Lett. 100, 045004 (2008).

[11] P. Gibbon and E. Forster, Plasma Phys. Controlled Fusion 38, 769 (1996).

[12] T. Caillaud, F. Blasco, F. Dorchies, Y. Glinec, Ch. Stenz, and J. Stevefelt, Nucl. Instrum. Methods Phys. Res., Sect. B 205, 329 (2003).

[13] R. C. Issac, G. Vieux, B. Ersfeld, E. Brunetti, S.P. Jamison, J. Gallacher, D. Clark, and D. A. Jaroszynski, Phys. Plasmas 11, 3491 (2004).

[14] O.F. Hagena and W. Obert, J. Chem. Phys. 56, 1793 (1972).

[15] N. L. Kugland et al., Appl. Phys. Lett. 92, 241504 (2008).

[16] L. M. Chen et al., Appl. Phys. Lett. 90, 211501 (2007).

[17] M. Kundu and D. Bauer, Phys. Rev. Lett. 96, 123401 (2006); T. Taguchi et al., Phys. Rev. Lett. 92, 205003 (2004); C. Jungreuthmayer et al., Phys. Rev. Lett. 92, 133401 (2004).

[18] C. Hombourger, J. Phys. B 31, 3693 (1998).

[19] K. D. Sevier, Gy. Szabó, and F. Folkmann, J. Phys. B 14, 4065 (1981).

[20] T. Ditmire et al., Phys. Rev. A 53, 3379 (1996). 